\title{
Porta de entrada para o diagnóstico da tuberculose: avaliação da estrutura dos serviços
}

\author{
Entryway for tuberculosis diagnosis: an assessment of service structure
}

Puerta de entrada al diagnóstico de tuberculosis: evaluación de la estructura de los servicios

Priscila Balderrama ${ }^{1}$, Silvia Figueiredo Vendramini ${ }^{2}$, Maria de Lourdes Sperli Geraldes Santos ${ }^{3}$, Maria Amélia Zanon Ponce ${ }^{4}$, Isabela Cristina Oliveira ${ }^{5}$, Tereza Cristina Scatena Villa ${ }^{6}$, Anneliese Domingues Wysocki ${ }^{7}$, Rubia Laine de Paula Andrade ${ }^{8}$

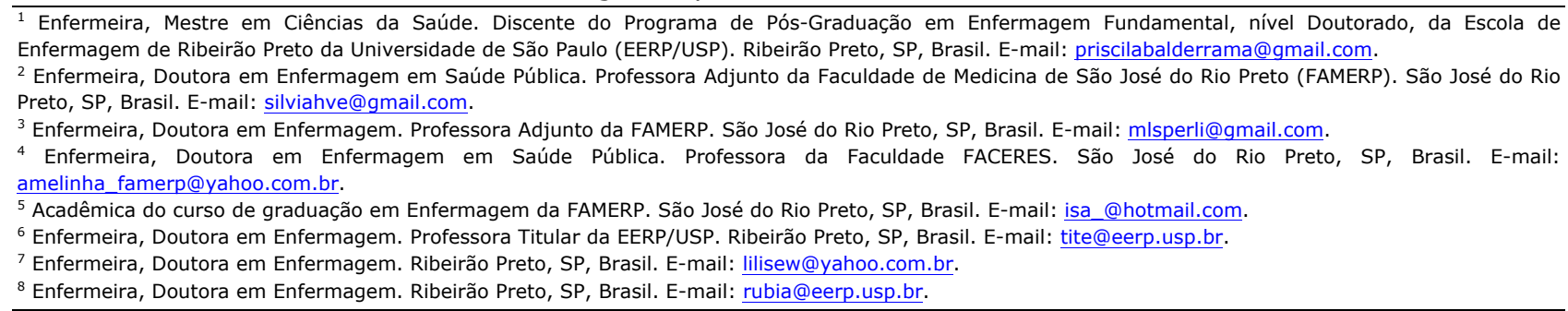

\section{RESUMO}

Estudo quantitativo em que foram entrevistados 160 profissionais em Unidades Básicas, Saúde da Família e Pronto Atendimento, com o objetivo de avaliar a estrutura dos serviços que atuam como porta de entrada para o diagnóstico da tuberculose. Como resultado, observou-se predomínio de profissionais do sexo feminino e mediana de tempo de trabalho superior nas Unidades Básicas. O Pronto Atendimento foi mais bem avaliado em horário de funcionamento e pior em disponibilidade de funcionários. A Saúde da Família apresentou melhor avaliação em tempo de espera para atendimento dos usuários e rotatividade de profissionais. Todos apresentaram resultados satisfatórios para insumos, mas nenhum possuía Raio-X. De maneira geral, embora as Unidades Básicas e de Saúde da Família tenham apresentado melhor avaliação, não se constituem como porta de entrada preferencial para o usuário que, na iminência dos sintomas, procura mais pelo Pronto-Atendimento.

Descritores: Tuberculose; Atenção Primária à Saúde; Avaliação de Serviços de Saúde.

\section{ABSTRACT}

A quantitative study which interviewed 160 professionals from Basic Health Units, Family Health and Emergency Services. The objective was to evaluate the structure of services that act as an entryway for diagnosing tuberculosis. We observed a prevalence of female professionals with superior median time of work in the Basic Units. Emergency Services received the highest evaluation regarding working hours and the lowest regarding staff availability. Family Health presented the best evaluation with respect to user waiting time and staff turnover. All services presented satisfactory results with respect to materials, but none had X-ray equipment. Generally speaking, although the Basic Units and Family Health services received the best evaluations, they do not constitute the preferred user entryway. In the imminence of symptoms, users seek out primarily Emergency Services.

Descriptors: Tuberculosis; Primary Health Care; Health Services Evaluation.

\section{RESUMEN}

Estudio cuantitativo en el que fueron entrevistados 160 profesionales en Unidades Básicas, Salud de la Familia y Pronto Socorro, objetivándose evaluar la estructura de los servicios que actúan como puerta de entrada al diagnóstico de la tuberculosis. Como resultado, se observó predominio de profesionales de sexo femenino y mediana de tiempo de trabajo superior en las Unidades Básicas. El Pronto Socorro resultó mejor evaluado en horario de funcionamiento y peor ante escasez de empleados. La Salud de la Familia presentó mejor evaluación en tiempo de espera para atención de usuarios y rotación de profesionales. Todos expresaron resultados satisfactorios para insumos, pero ninguno contaba con unidad de Rayos X. De modo general, aunque las Unidades Básicas y de Salud de la Familia hayan presentado mejor evaluación, no se constituyen como puerta de entrada preferencial para el paciente que, urgido por los síntomas, prefiere atenderse en el Pronto Socorro.

Descriptores: Tuberculosis; Atención Primaria de Salud; Evaluación de Servicios de Salud. 


\section{INTRODUÇÃo}

Nos sistemas de saúde orientados por uma política de atenção primária à saúde (APS), a porta de entrada corresponde ao primeiro contato entre o usuário e o serviço de saúde. Deve, por essa razão, ser de fácil acesso para que a atenção seja adequada, oportuna, resolutiva e, ainda, assegure, quando necessário, a continuidade da atenção em outros pontos do sistema com maior concentração tecnológica, para atendimento integral às necessidades de saúde do usuário ${ }^{(1-2)}$.

No processo de consolidação do sistema público de saúde brasileiro, a Atenção Básica $(A B)$ foi concebida para se tornar o eixo estruturante e a porta de entrada

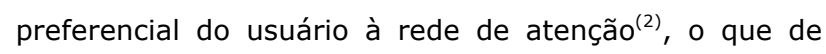
fato não tem ocorrido e, por conseguinte, leva os usuários a buscarem por outros serviços de saúde, em especial, os de Pronto-Atendimento (PA $)^{(2-3)}$.

Esse descompasso pode ser caracterizado, em partes, pelas evidentes barreiras de acesso relacionadas à $A B$ (ausência de profissionais, recursos materiais e diagnósticos, lógica territorial inflexível, limitação no horário de funcionamento e ausência de organização para atendimento da demanda programática e espontânea $)^{(2-3)}$. Soma-se a isso a estruturação ineficiente do sistema de saúde como um todo, que, embora conviva com uma situação epidemiológica caracterizada pela tripla carga de doenças (infecciosas, crônicas e causas externas), orienta-se, principalmente, para o atendimento das condições agudas e agudização das crônicas, em detrimento do fortalecimento da rede de atenção ${ }^{(2,4)}$.

Nesse cenário, insere-se a Tuberculose (TB), considerada pela Organização Mundial de Saúde (OMS) uma condição crônica e um grave problema de saúde pública ${ }^{(5)}$. Conhecer a estrutura oferecida pelos serviços de saúde para o diagnóstico da TB, especialmente aqueles que se constituem como "portas de entrada", pode facilitar a implementação de medidas que favoreçam o melhor desempenho dos serviços e possibilite a redução dos índices de morbimortalidade associados à doença.

A normatização referente às "portas de entrada" é recente no Brasil. Disposta no Decreto no 7.508, de 28 de junho de 2011, nela tal conceito é definido como "serviços de atendimento inicial à saúde do usuário"(6). Os serviços de urgência e emergência são regulamentados como portas de entrada nas Redes de Atenção à Saúde no SUS, juntamente com as Unidades Básicas, cuja importante missão é, integrado à rede de atenção, ser co-responsável por um dos atributos da $A B$, o acesso de primeiro contato ${ }^{(1)}$.

Figura 1: O Sistema de Serviços de Saúde.

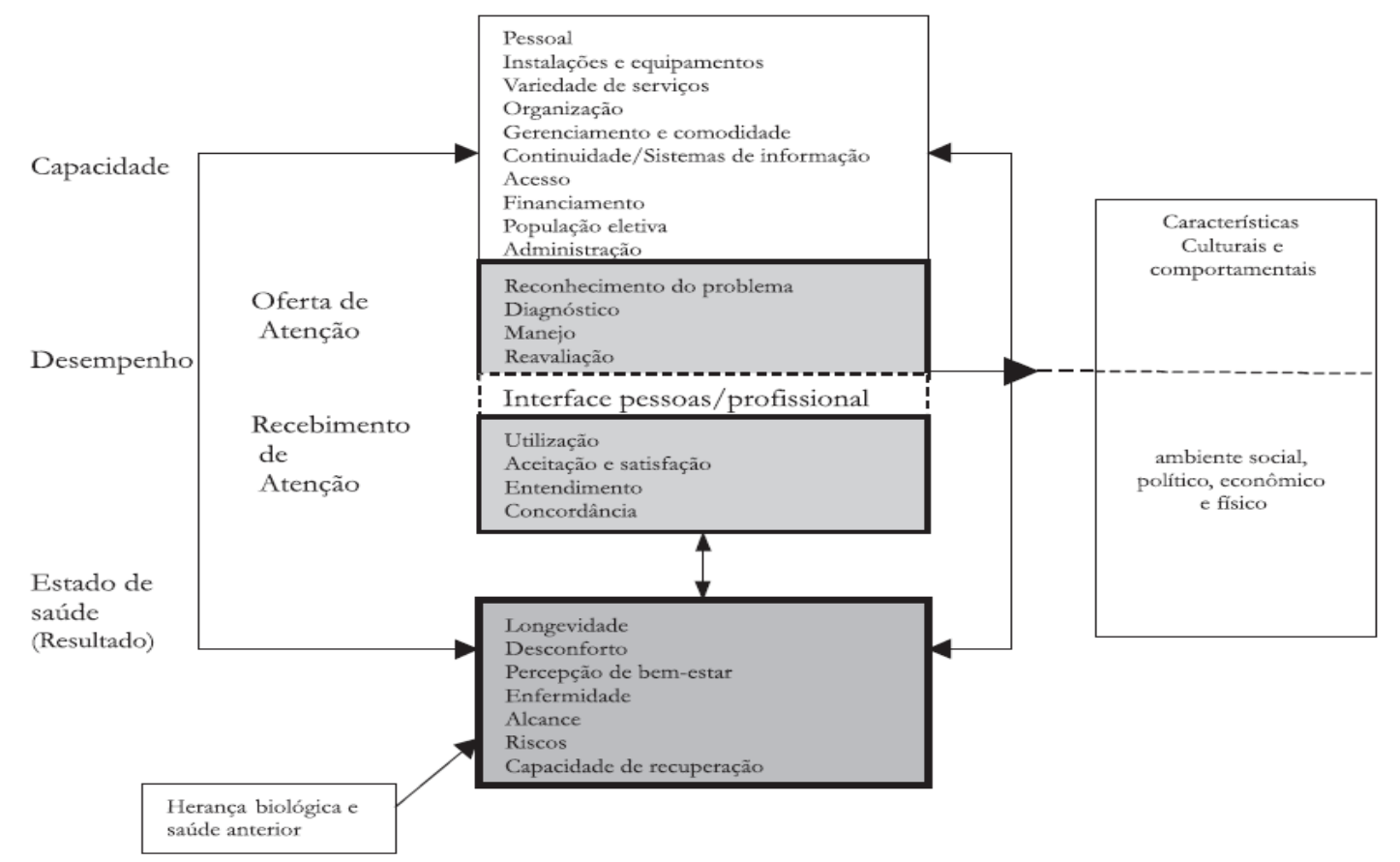

Fonte: Starfield ${ }^{(1)}$.

A reformulação do sistema de saúde para atendimento dessa nova diretriz precisa considerar aspectos de necessidade de saúde da população, da oferta de serviços, além de considerar, de modo 
abrangente, tecnologias e recursos necessários à atenção $^{(3)}$. Nesse sentido, a avaliação constitui-se ferramenta útil ao processo de gestão, estando, inclusive, presente nas atuais diretrizes políticas, que preconizam sua aplicação como ferramenta de melhoria dos serviços de saúde ${ }^{(6)}$.

Para realização da avaliação proposta neste estudo, utilizou-se como referencial teórico a proposta de avaliação do desempenho dos serviços de saúde de Starfield, baseada nos componentes do sistema de saúde: estrutura, processo e resultado, propostos por Donabedian $^{(7)}$, para o qual cada sistema de serviços de saúde possui uma estrutura (ou capacidade) de oferta de serviços; os processos (ou desempenho), que envolvem as ações dos profissionais e pacientes, e os resultados, relacionados ao estado de saúde obtido a partir da assistência prestada(1).

Assim, diante da importância do diagnóstico precoce da TB e da necessidade de avaliar constantemente os serviços de saúde, em especial os que atuam como "porta de entrada", concebeu-se este estudo, visando identificar os aspectos relacionados à estrutura dos serviços que podem colaborar no retardo do diagnóstico, em um município prioritário para o controle da TB no Brasil. Além disso, poucos são os artigos que tratam da avaliação de serviços ou programas de saúde, embora esta seja uma nova diretriz da política de saúde brasileira.

Reconhece-se, portanto, que o desempenho das ações de controle da TB está sujeito à estrutura ofertada e demanda a realização de estudos que permitam avaliar os fatores estruturais envolvidos no acesso ao diagnóstico, a fim de se obter subsídios para o planejamento de políticas de intervenção e manejo da doença.

\section{MÉTODOS}

Trata-se de um estudo epidemiológico, descritivo e exploratório, do tipo inquérito, realizado em Unidades Básicas de Saúde (UBS), Unidades Básicas de Saúde da Família (UBSF) e Unidades de Pronto Atendimento (UPA) em um município do interior do Estado de São Paulo.

A população do estudo foi composta por médicos, enfermeiros, técnicos e auxiliares de enfermagem que atuavam nas referidas instituições de saúde. Para cálculo da amostra, inicialmente, realizou-se o levantamento do número de UBSs, UBSFs e UPAs existentes no município. Em seguida, levantou-se o número de profissionais que atuavam na rede básica e pública do município em 2009, tendo sendo excluídas da amostra as duplicidades e aqueles que trabalham predominantemente em estabelecimentos hospitalares e clínicas particulares. A população do estudo foi composta por 571 profissionais de saúde distribuídos em nas UBS, UBSF e UPA.

A amostra de profissionais de saúde entrevistados foi calculada assumindo variância média dos estratos $S d^{2}=1$ para as UBSF, valor proposto na literatura por Almeida e Macinko(8) e de $S d^{2}=1,2$ para as UBS e UPA e a diferença entre a média amostral simples dos estratos e a média da população ( $\beta=0,23$ ) e probabilidade do erro tipo I igual a $\left(\mathrm{Z}_{\alpha}=1,96\right)$, tem-se a equação $n=S d^{2}\left(Z_{\alpha}\right)^{2} / \beta^{2}$ (9). Assumindo $20 \%$ de perdas, a amostra final calculada foi para 160 profissionais.

Com o objetivo de analisar grupos mais homogêneos, para comparação entre estratos, optou-se por entrevistar 54 profissionais de cada tipo de serviço de saúde. Realizou-se, em seguida, a proporção por categoria profissional que foi dividida pelo total de profissionais contabilizados no município, sendo entrevistados 25 médicos, sete enfermeiros e 22 auxiliares/técnicos em cada tipo de serviço.

A coleta dos dados ocorreu no período de Julho a Outubro de 2009 por meio de entrevista com os profissionais de cada tipo de serviço. As Unidades de Saúde, onde foram realizadas as entrevistas, foram escolhidas aleatoriamente, por meio de sorteio. Esgotadas todas as possibilidades de entrevista em uma determinada Unidade, posteriormente, dirigiu-se à outra.

Para realização da entrevista, utilizou-se um questionário estruturado, elaborado a partir do referencial da Atenção Primária em Saúde (APS), elencando as dimensões porta de entrada, acesso, elenco de serviços, coordenação, orientação à comunidade e enfoque na família(1).

Os entrevistados responderam a cada pergunta do questionário com respostas dicotômicas (Sim ou Não), abertas, ou segundo uma escala de possibilidades preestabelecida, escala de Likert, à qual foi atribuído um valor entre um e cinco, que permitiu registrar o grau de relação de preferência (ou concordância) das afirmações. Em algumas questões, o valor zero foi atribuído para resposta não sei e o valor 99 para a opção não se aplica.

Foram criados indicadores de estrutura, sendo que, para as perguntas com respostas dicotômicas, considerou-se como indicador a proporção de ocorrência de cada item avaliado. Estes indicadores foram 
analisados individualmente e comparados entre diferentes unidades de saúde, a partir dos intervalos de confiança das proporções dos indicadores criados.

Para as questões com escala Likert de resposta (valores de um a cinco), o indicador correspondeu ao valor médio de todas as respostas dos profissionais de saúde ao item avaliado. Neste caso, o resultado foi classificado segundo os valores obtidos dos indicadores: aos valores próximos de 1 e 2, foi estipulada a classificação não satisfatório, próximo de 3 , regular, e próximo de 4 e 5, satisfatório. Para as questões com leitura inversa da escala Likert de resposta (valores de 5 a 1), com valores obtidos próximos de 1 e 2, foi considerada a classificação satisfatório, próximo de 3 , regular, e próximo de 4 e 5, não satisfatório.

Para este estudo, de acordo com o referencial teórico e questionário adotados, consideraram-se as seguintes variáveis:

- caracterização dos sujeitos: sexo e tempo de atuação no serviço;

- variáveis que permitiram identificar aspectos relacionados à estrutura dos serviços, descritos no Quadro 1:

Quadro 1. Variáveis levantadas para identificar aspectos relacionados ao componente estrutura, da avaliação dos serviços de saúde:

\begin{tabular}{|c|c|}
\hline \multirow{2}{*}{$\begin{array}{c}\text { CAPACIDADE }= \\
\text { ESTRUTURA }\end{array}$} & $\begin{array}{c}\text { Pessoal: rotatividade de recursos humanos, disponibilidade suficiente de profissionais } \\
\text { no serviço de saúde, percepção dos profissionais quanto à falta de conhecimento da } \\
\text { doença e à existência de sobrecarga de trabalho. }\end{array}$ \\
\cline { 2 - 3 } & $\begin{array}{c}\text { Instalações e equipamentos: adequação do local destinado para a coleta de escarro } \\
\text { no serviço de saúde, disponibilidade suficiente de pote para coleta de escarro, geladeira } \\
\text { para o acondicionamento de material biológico, formulário para pedido de baciloscopia e } \\
\text { sala de raio-x na Unidade. }\end{array}$ \\
\cline { 2 - 3 } & $\begin{array}{c}\text { Organização: horário de funcionamento do serviço de saúde que o profissional } \\
\text { trabalha. }\end{array}$ \\
\cline { 2 - 3 } & Acesso: tempo em que os usuários levam para serem atendidos. \\
\hline
\end{tabular}

Os indicadores obtidos por meio da aplicação do questionário foram analisados individualmente e comparados entre os três tipos de serviço, utilizando-se a análise de variância (ANOVA), com o uso do teste $F$, e a comparação múltipla entre médias por meio da aplicação do teste de Tukey. O nível de significância estatística adotado em todos os testes foi de $5 \%$ de probabilidade.

Esta pesquisa foi previamente submetida e aprovada pelo Comitê de Ética em Pesquisa da Faculdade de Medicina de São José do Rio Preto-SP, conforme Protocolo no 1212/2009 e parecer n042/2009, atendendo às recomendações éticas preconizadas.

\section{RESULTADOS}

Foram entrevistados os 54 profissionais nas UBS e UPA e 52 profissionais nas UBSF. Encontrou-se uma predominância do sexo feminino entre os profissionais de saúde em todos os tipos de serviços ( $81,2 \%$ nas UBS; $84,6 \%$ nas UBSF e $63 \%$ na UPA).

A mediana de tempo de trabalho nos serviços municipais de saúde para os profissionais das UBS foi de nove anos, variando de 1,4 a 28 anos. Para os profissionais das UBSF, a mediana de tempo de trabalho nos serviços municipais de saúde foi de 6,5 anos (variação de 0,6 a 31 anos) e, para os profissionais da UPA, foi de cinco anos, variando de 0,08 a 30 anos.

Em relação à avaliação da estrutura, na Tabela 1, são apresentados os resultados de média, desvio-padrão e o valor do teste $F$ para os sete indicadores de estrutura de acordo com os três grupos de serviços de saúde. A Tabela 2 apresenta 0 resultado dos testes de comparação múltipla (teste de Tukey) entre os indicadores nos três grupos.

Os indicadores: horário de funcionamento, rotatividade de recursos humanos $(R H)$, disponibilidade de profissionais de saúde, percepção quanto à sobrecarga de trabalho e tempo de espera dos usuários para serem atendidos foram diferentes entre os três grupos de serviços de saúde (Tabela 1).

Verificou-se que, embora os três tipos de serviços tenham apresentado indicadores satisfatórios em relação ao horário de funcionamento, a UPA foi o serviço que teve o melhor indicador (Tabela 1), diferenciando-se das UBS e UBSF (Tabela 2). A UPA também se distinguiu das UBS e UBSF (Tabela 2) em relação ao indicador disponibilidade de profissionais de saúde, apresentando pior resultado (Tabela 1 ). 
Tabela 1: Indicadores da estrutura dos serviços de saúde, segundo a média por tipo de serviço na percepção dos profissionais de saúde. São José do Rio Preto, SP, Brasil, 2009.

\begin{tabular}{|c|c|c|c|c|c|c|c|c|c|c|}
\hline \multirow[t]{2}{*}{ Indicadores } & \multicolumn{3}{|c|}{$\begin{array}{l}\text { Unidade Básica de } \\
\text { Saúde }\end{array}$} & \multicolumn{3}{|c|}{$\begin{array}{l}\text { Unidade de Saúde da } \\
\text { Família }\end{array}$} & \multicolumn{3}{|c|}{$\begin{array}{l}\text { Unidade Pronto- } \\
\text { Atendimento }\end{array}$} & \multirow[t]{2}{*}{$\mathrm{p}$} \\
\hline & $\mathbf{n}$ & média & dp & $\mathbf{n}$ & média & dp & $\mathbf{n}$ & média & dp & \\
\hline Horário de funcionamento ${ }^{a}$ & 54 & 4,0 & 0,7 & 52 & 4,1 & 0,7 & 54 & 4,5 & 0,9 & 0,0056 \\
\hline $\begin{array}{c}\text { Rotatividade de recursos } \\
\text { humanos }\end{array}$ & $53 *$ & 2,8 & 1,1 & $49 *$ & 2,3 & 0,9 & 54 & 3,1 & 1,0 & 0,0009 \\
\hline $\begin{array}{l}\text { Disponibilidade de } \\
\text { profissionais de saúde }\end{array}$ & $53 *$ & 2,8 & 1,2 & 52 & 3,1 & 1,4 & 54 & 2,3 & 1,0 & 0,0030 \\
\hline $\begin{array}{l}\text { Usuários demoram para } \\
\text { serem atendidos }{ }^{c}\end{array}$ & $50 *$ & 3,3 & 1,1 & 52 & 4,0 & 1,1 & 54 & 2,9 & 1,0 & $<0,0001$ \\
\hline Falta de conhecimento sobre & & & & & & & & & & \\
\hline $\begin{array}{l}\text { TB pelos profissionais de } \\
\text { saúde }^{\text {b }}\end{array}$ & $53 *$ & 3,7 & 1,0 & $51 *$ & 3,7 & 1,1 & 54 & 3,6 & 1,1 & 0,8539 \\
\hline $\begin{array}{c}\text { Percepção quanto à } \\
\text { sobrecarga de trabalho }\end{array}$ & $53 *$ & 2,6 & 1,5 & $51 *$ & 3,5 & 1,5 & 54 & 2,6 & 1,4 & 0,0007 \\
\hline $\begin{array}{c}\text { Adequação do local destinado } \\
\text { para coleta de escarro }\end{array}$ & $38 *$ & 2,7 & 0,9 & $38 *$ & 2,3 & 1,1 & $15^{*}$ & 2,7 & 0,8 & 0,3947 \\
\hline \multicolumn{11}{|c|}{$\begin{array}{l}\text { Escalas de respostas: } \\
\text { a } 1 \text { - muito ruim; } 2 \text {-ruim; } 3 \text { - regular; } 4 \text { - bom; } 5 \text { - muito bom; } \\
\text { b } 1 \text { - nunca; } 2 \text { - quase nunca; } 3 \text { - às vezes; } 4 \text { - quase sempre; } 5 \text { - sempre. } \\
\text { c } 1 \text { - sempre; } 2 \text { - quase sempre; } 3 \text { - às vezes; } 4 \text { - quase nunca; } 5 \text { - nunca. } \\
\text { - Excluídos os profissionais que responderam não sei e não se aplica } \\
\text { Em negrito } p<0,05 \text { - diferença estatística }\end{array}$} \\
\hline
\end{tabular}

Tabela 2. Comparação dos indicadores de estrutura entre as Unidades Básicas de Saúde, Unidades Básicas de Saúde da Família e Unidades de Pronto-Atendimento,

segundo a percepção dos profissionais de saúde. São José do Rio Preto, SP, Brasil, 2009.

\begin{tabular}{|c|c|c|c|c|}
\hline \multirow{2}{*}{ Indicadores } & \multicolumn{3}{|c|}{ Grupos de comparação } & \multirow{2}{*}{ Conclusão } \\
\hline & UBS $x$ UBSF & UBS $\times$ UPA & UPA $\times$ UBSF & \\
\hline \multirow{4}{*}{ Horário de funcionamento ${ }^{a}$} & & & & UBS $=$ UBSF \\
\hline & 0,959 & 0,0083 & 0,0208 & UBS $\neq$ UPA \\
\hline & & & & UBSF $\neq$ UPA \\
\hline & & & & UBS $=$ UBSF \\
\hline \multirow[t]{3}{*}{ Rotatividade de $\mathrm{RH}^{\mathrm{b}}$} & 0,0835 & 0,1961 & 0,0004 & UBS = UPA \\
\hline & & & & UBSF $\neq$ UPA \\
\hline & & & & UBS $=$ UBSF \\
\hline \multirow[t]{3}{*}{ Disponibilidade de profissionais de saúde ${ }^{b}$} & 0,5508 & 0,049 & 0,0021 & UBS $\neq$ UPA \\
\hline & & & & UBSF $\neq$ UPA \\
\hline & & & & UBS $\neq$ UBSF \\
\hline \multirow[t]{3}{*}{ Usuários demoram para serem atendidos ${ }^{c}$} & 0,0028 & 0,0832 & $<0,0001$ & UBS = UPA \\
\hline & & & & UBSF $\neq$ UPA \\
\hline & & & & UBS $\neq$ UBSF \\
\hline \multirow[t]{2}{*}{ Percepção quanto à sobrecarga de trabalhob } & 0,002 & 0,9995 & 0,0021 & UBS = UPA \\
\hline & & & & UBSF $\neq$ UPA \\
\hline
\end{tabular}

Escalas de respostas:

a 1 - muito ruim; 2 - ruim; 3 - regular; 4 - bom; 5 - muito bom;

b 1 - nunca; 2 - quase nunca; 3 - às vezes; 4 - quase sempre; 5 - sempre.

c 1 - sempre; 2 - quase sempre; 3 - às vezes; 4 - quase nunca; 5 - nunca.

Em negrito $p<0,05$ - diferença estatística

Legenda:

UBS - Unidades Básicas de Saúde;

UBSF - Unidade Básica de Saúde da Família;

UPA -Unidade de Pronto-Atendimento

As UBSF mostraram melhor avaliação no tempo de espera dos usuários para serem atendidos (Tabela 1), diferenciando-se das UBS e UPA (Tabela 2). As UBSF também apresentaram uma menor rotatividade dos profissionais, com avaliação satisfatória (Tabela 1) e diferenciaram-se da UPA (Tabela 2), avaliada como insatisfatório (Tabela 1 ). Já em relação à percepção quanto à sobrecarga de trabalho, as UBSF apresentaram desempenho insatisfatório (Tabela 1), diferindo-se das UBS e UPA (Tabela 2), que foram mais bem avaliadas.

Não se encontrou diferença estatística entre os grupos para falta de conhecimento sobre TB pelos profissionais de saúde. Verificou-se que, nos três tipos de serviços, quase nunca se observa falta de conhecimento sobre a TB, segundo a percepção dos entrevistados, sendo este indicador avaliado próximo de satisfatório (Tabela 1 ). 
Também não se encontrou diferença que fosse estatisticamente significante entre os grupos para 0 indicador adequação do local para coleta de escarro, cuja avaliação foi insatisfatória nos três grupos, visto que muitos não possuíam um local apropriado para a coleta, resultando na opção de resposta "não se aplica".

Em relação aos insumos e materiais disponíveis, não foram encontradas diferenças estatísticas entre os três tipos de serviços. De acordo com os profissionais entrevistados, $94,0 \%$ das UBS possuem formulário para solicitar baciloscopia, 53,0\% pote de escarro e 96,0\% geladeira para armazenar material biológico. Nas UBSF, $86,6 \%$ dos profissionais referiram à disponibilidade de formulário para solicitar baciloscopia, 53,8\% de pote de escarro e $90,4 \%$ de geladeira. Já os profissionais entrevistados na UPA, 70,3\% apontaram disponibilidade de formulário para solicitar baciloscopia, $75,9 \%$ de pote de escarro e geladeira. Nenhuma Unidade de Saúde possuía Raio-X.

Tabela 3: Proporção e intervalo de confiança dos insumos e equipamentos disponíveis nos serviços de saúde para o diagnóstico da tuberculose, segundo os profissionais de saúde. São José do Rio Preto, SP, Brasil, 2009.

\begin{tabular}{|c|c|c|c|c|c|c|}
\hline \multirow{2}{*}{$\begin{array}{l}\text { Insumos e equipamentos para } \\
\text { exames }\end{array}$} & \multicolumn{2}{|c|}{$\begin{array}{l}\text { Unidades Básicas de } \\
\text { Saúde }(n=54)\end{array}$} & \multicolumn{2}{|c|}{$\begin{array}{l}\text { Unidades Saúde da } \\
\text { Família }(n=52)\end{array}$} & \multicolumn{2}{|c|}{$\begin{array}{l}\text { Pronto Atendimento } \\
\qquad(n=54)\end{array}$} \\
\hline & $\%$ & IC & $\%$ & IC & $\%$ & IC \\
\hline $\begin{array}{c}\text { Proporção de serviços com formulário } \\
\text { para solicitar baciloscopia }\end{array}$ & 94 & $(88-100)$ & 87 & $(77-96)$ & 70 & $(58-83)$ \\
\hline $\begin{array}{c}\text { Proporção de serviços com pote de } \\
\text { escarro }\end{array}$ & 54 & $(40-67)$ & 54 & $(40-67)$ & 76 & $(65-87)$ \\
\hline Proporção de serviços com geladeira & 96 & $(91-100)$ & 90 & $(82-98)$ & 76 & $(65-87)$ \\
\hline Proporção de serviços com raio-X & 0 & - & 0 & - & 0 & - \\
\hline
\end{tabular}

\section{DISCUSSÃO}

No município estudado, o usuário suspeito de TB tem como porta de entrada, no sistema municipal de saúde, as UBSs, UBSFs ou UPAs. Entretanto, estudos recentes $^{(10-11)}$ confirmam que o serviço hospitalar ainda é o principal responsável pelo diagnóstico da doença, seguido pela atenção básica. Nesse contexto, em relação ao item "horário de funcionamento", existem indicativos ${ }^{(11-12)}$ de que um período de atendimento mais compatível às necessidades do usuário poderia facilitar seu acesso, contrariamente à avaliação positiva realizada pelos profissionais de saúde para esse item. O mesmo ocorreu em relação ao tempo em que os usuários levam para serem atendidos. Sua avaliação positiva não corresponde aos resultados de outros estudos, em que os sujeitos entrevistados foram os doentes ${ }^{(10,12)}$.

Contudo, observa-se que a dificuldade de acesso do paciente, e consequente demora para efetivação do diagnóstico da TB, não é uma exclusividade do município, uma vez que fato semelhante foi constatado em diferentes regiões no Brasil ${ }^{(12)}$ e do mundo ${ }^{(13)}$.

Ademais, essa "dificuldade" de acesso também não é fato restrito à TB, e pode-se explicar, dentre outros motivos, pela atual incoerência dos sistemas fragmentados de saúde, que, mesmo com predomínio de doenças crônicas, são voltados, principalmente, para o tratamento das condições agudas e agudizações das condições crônicas ${ }^{(4)}$.
A situação epidemiológica da população brasileira, não diferente da situação ora apresentada, aponta para a tripla carga de doenças, que envolve, ao mesmo tempo, uma agenda não concluída de doenças agudas, alto índice de doenças crônicas e também o forte crescimento das causas externas ${ }^{(4)}$. Logo, para uma melhor inserção do usuário nesse sistema, constata-se a necessidade iminente não só de uma atenção sistemática e planejada, como também do atendimento à demanda espontânea, principalmente nas unidades ambulatoriais e de pronto-atendimento ${ }^{(2)}$.

Sobre rotatividade de recursos humanos, os resultados obtidos neste trabalho ratificam inúmeros outros estudos que apontam para uma acentuada rotatividade de profissionais de saúde em instituições públicas, $^{(14-15)}$ fato que dificulta a continuidade e a integralidade do cuidado.

No Brasil, muito tem se discutido sobre as inúmeras modalidades de contratação de recursos humanos dentro do mesmo serviço de saúde e os diferentes salários para o exercício da mesma função, o que parece colaborar para que aconteça tal rotatividade, inclusive no município de estudo.

Dentre os fatores responsáveis por essa situação, podem-se citar a Lei Complementar no 101 de 04 de maio de $2000^{(16)}$, popularmente conhecida por Lei de Responsabilidade Fiscal, que limita os gastos públicos com funcionários, e o próprio histórico de efetivação do SUS, com a descentralização da saúde e incorporação de 
profissionais com diferentes vínculos dentro da mesma esfera de governabilidade ${ }^{(17)}$.

Embora todas essas situações favoreçam a não fixação de alguns profissionais e também o descontentamento de muitos outros, atualmente, algumas alternativas legais podem priorizar a qualidade e melhoria dos serviços de saúde para o paciente, associada a melhores condições de trabalho para o profissional de saúde. Como exemplo, pode-se citar as modalidades de contrato de gestão, em que se preconiza a gestão por metas quanti e qualitativas, a avaliação e o monitoramento dos indicadores de saúde ${ }^{(16,18)}$, bem como as garantias de promoção e progressão salarial por desempenho e direitos trabalhistas assegurados aos profissionais de saúde.

No Brasil, essa modalidade de contratação iniciou-se com o advento das Organizações Sociais de Saúde (OSS), concebidas no decorrer da Reforma da Administração Pública ${ }^{(16)}$. Atualmente, embora alvo de muitas críticas, as avaliações em torno das OSS têm sido altamente positivas ${ }^{(19)}$, culminando com hospitais mais produtivos, de melhor qualidade assistencial e mais baratos $^{(20)}$.

Estudos indicam que os bons resultados dependem da adequada escolha do parceiro com experiência gerencial, que faça integração com as demais Unidades da Rede Pública de Saúde e seja fortemente acompanhado e fiscalizado tanto pelo poder público como pelo controle social( ${ }^{(14)}$. É imprescindível, acima de tudo, que seja antecedido pela prévia elaboração do Contrato de Gestão, cujas metas devem ser claras e de fácil quantificação(18).

No que diz respeito à disponibilidade de profissionais no serviço de saúde, os resultados deste trabalho condizem com aqueles encontrados em outros estudos, segundo os quais há debilidade quantitativa de recursos humanos ${ }^{(21)}$, resultado compatível ao constatado no município de Ribeirão Preto-SP, onde se observou que os Pronto-Atendimentos apresentavam maior rotatividade de profissionais e menor quantidade de profissionais disponíveis em relação às $U_{B S s^{(15)}}$. Já em relação à sobrecarga de trabalho, as UBSF contraditoriamente apresentaram pior desempenho, diferenciando-se das UBSs e UPAs, mais bem avaliadas.

Reconhece-se que a ausência de funcionários pode levar à sobrecarga de trabalho. Porém, esse estudo mostrou haver uma contradição, uma vez que as UBSF apresentam maior disponibilidade de recursos humanos e, ainda assim, maior sobrecarga de trabalho. Em saúde, contudo, esse fato pode não estar associado apenas à quantidade, mas também à ausência de treinamento e falta de RH qualificado, além da ausência de políticas que avaliem o desempenho profissional e da falta de motivação e adequada supervisão ${ }^{(21)}$.

Quanto à falta de conhecimento sobre a TB, os três tipos de serviços foram avaliados positivamente, o que também aconteceu no município de Ribeirão Preto-SP em estudo semelhante, de acordo com o qual profissionais de saúde foram igualmente abordados sobre conhecimento em relação à $\mathrm{TB}^{(15)}$. Estudo recentemente realizado no município de São José do Rio Preto-SP, no entanto, demonstrou o contrário(22).

Convém destacar que inúmeros trabalhos indicam a preocupação com a formação de recursos humanos para a atenção básica e mostram que as alternativas para resolução do impasse vão muito além das possibilidades do setor saúde. É premente, para esse fim, discussão intersetorial(23) $^{(23)}$ priorize não somente a formação de profissionais com perfil generalista durante a graduação, mas também a ampliação das vagas em residências e cursos de pós-graduação voltados para a atenção primária. Além disso, deve ser destacada a importância da qualificação dos profissionais que atuam no prontoatendimento, tendo em vista a grande importância deste serviço na configuração das Redes de Atenção à Saúde ${ }^{(6)}$.

Considerando a inadequação da formação profissional, e, partindo do princípio que a sua readequação, mesmo que priorizada politicamente, não se efetivaria em um curto período de tempo, a solução mais viável para o problema, seria a criação de espaços de discussão in loco, com supervisões contínuas, a fim de identificar as falhas e dificuldades no processo para investir-se no aprimoramento das habilidades adquiridas $^{(21)}$.

No que tange à estrutura física, os resultados do estudo apontaram para a inexistência de local adequado à coleta do material biológico nas Unidades de Saúde, outrora realizada nos domicílios e ambientes externos das Unidades. Entretanto, sabe-se que esta situação não é exclusiva do município e vai ao encontro da recomendação do MS, que orienta a realização da coleta em local aberto, preferencialmente ao ar livre ${ }^{(24)}$.

Em relação aos impressos, cabe destacar que um número significativo de profissionais das UPAs relatou não haver disponibilidade em quantidade suficiente, fato que não condizia com a realidade. Outra opção de resposta foi "não saber" responder, como ocorreu principalmente em UBSFs e UPAs. Curiosamente, a análise da mediana do tempo de trabalho nestes dois 
tipos de serviços de saúde foi inferior à UBS, onde a quase totalidade dos profissionais tiveram respostas assertivas.

No que se refere à existência de pote para coleta de escarro, verificou-se que todas as Unidades apresentavam o insumo supracitado. Contudo, em relação à existência de geladeira para o acondicionamento de material biológico, foi possível constatar que não havia uma própria para o acondicionamento deste material em nenhuma das Unidades pesquisadas e a geladeira em questão tratavase da mesma onde eram acondicionados o sangue e outros materiais. Vale ressaltar que o material biológico (escarro) usualmente é trazido pelo paciente à Unidade no período matutino e acondicionado em isopor, procedimento adequado de acordo com as normas do MS para acondicionamento ${ }^{(24)}$.

No que se refere à existência de sala de Raios- $X$, a grande maioria dos entrevistados relatou inexistência do equipamento, ao contrário do observado no município de Ribeirão Preto-SP, onde as Unidades de Pronto Atendimento possuíam o equipamento(15), embora a evolução da tuberculose seja acompanhada de anormalidades radiográficas na maioria dos casos, motivo pelo qual a radiografia do tórax é importante auxiliar no diagnóstico ${ }^{(23)}$.

O município estudado possui cinco ProntoAtendimentos, que, no momento da coleta de dados, receberam a denominação de Unidade de Pronto Atendimento - UPA 24 horas, em alusão a uma nova política de saúde, em concordância com a Portaria No 1020 , de 13 de maio de $2009^{(25)}$. De acordo com a referida Portaria, independente da classificação, todas as UPAs deveriam contar com uma sala de exames de radiologia geral, motivo pelo qual constatou-se que nenhuma das UPAs possuía a infraestrutura adequada e legalmente necessária, de acordo com a legislação vigente.

\section{REFERÊNCIAS}

1. Starfield $B$. Atenção primária: equilíbrio entre necessidades de saúde, serviços e tecnologia. Brasília: UNESCO/Ministério da Saúde; 2002.

2. Giovanella L, Mendonça MHM, Escorel S, Almeida PF, Fausto MCR, Andrade CLT et al. Potencialidades e obstáculos para a consolidação da Estratégia Saúde da Família em grandes centros urbanos. Saúde debate. 2010;34(85):248-64.

3. Oliveira LH, Mattos RA, Souza AIS. Cidadãos peregrinos: os "usuários" do SUS e os significados de sua demanda a prontossocorros e hospitais no contexto de um processo de reorientação do modelo assistencial. Cien Saude Colet [Internet]. 2009

\section{CONCLUSÃO}

De maneira geral, as UBSFs e UBSs têm melhor estrutura para diagnóstico da TB, por possuírem profissionais com maior tempo de trabalho nos serviços, menor rotatividade de recursos humanos, maior disponibilidade de funcionários, insumos necessários, além de fluxo adequado para transporte do material ao laboratório de referência. Entretanto, mesmo apresentando melhor estrutura que o ProntoAtendimento, estudos apontam que não são a porta de entrada preferencial do usuário, que, na iminência dos sintomas, procuram as UPAs mais bem avaliadas em relação ao horário de funcionamento. Por outro lado, mesmo sendo a porta de entrada preferencial do usuário, as UPAs não são as responsáveis pelo diagnóstico da doença, que, no município, acontece majoritariamente nos Hospitais.

Cabe salientar que para a Atenção Básica se tornar a porta de entrada preferencial do usuário e a responsável de fato pelo cuidado, alguns aspectos relacionados à gestão de recursos humanos e também à melhoria do acesso devem ser considerados. Impossível não destacar a necessidade de capacitação contínua dos funcionários e de discussão das modalidades de contratação, vínculos e fixação de profissionais. Da mesma maneira, não é mais possível discutir acesso, sem tratar da ampliação do horário de funcionamento das Unidades, que não é adequado à grande parte da população que trabalha e precisa de atendimento.

Uma vez que a capacitação contínua dos profissionais também esteja relacionada à capacidade de realização de diagnóstico, as UPAs mais mal avaliadas em rotatividade de funcionários, também necessitam adotar medidas que favoreçam a fixação. Sendo assim, além de se considerarem os aspectos organizacionais e de pessoal, há de se levar em conta também a questão de estrutura física, dada a ausência de equipamento básico para realização de diagnóstico, como a sala de Raio-X.

[acesso em: 30 set 2014];14 (5):1929-38. Disponível em: http://dx.doi.org/10.1590/S1413-81232009000500035. 4. Mendes EV. As redes de atenção à saúde. Cien Saude Colet [Internet]. 2010 [acesso em: 30 set 2014];15(5):2297-305. Disponível em: http://dx.doi.org/10.1590/S1413-

81232010000500005 .

5. Organização Mundial da Saúde. Cuidados inovadores para condições crônicas: componentes estruturais de ação: relatório mundial. Brasília: Organização Mundial da Saúde; 2003.

6. Secretaria de Gestão Estratégica e Participativa, Ministério da Saúde. Regulamentação da Lei 8.080 para fortalecimento do Sistema Único da Saúde: decreto 7508, de 2011. Rev Saude Publica [Internet]. 2011 [acesso em: 30 set 2014];45(6):1206- 
07. Disponível em: http://dx.doi.org/10.1590/S003489102011000600025.

7. Donabedian A. Evaluating the quality of medical care. The effectiveness of quality assurance. Int J Qual Health Care. 1996;8(4):401-7.

8. Almeida C, Macinko J. Validação de uma metodologia de avaliação rápida das características organizacionais e do desempenho dos serviços de atenção básica do Sistema Único de Saúde (SUS) em nível loca. Brasília: OPAS; 2006.

9. Bolfarine $\mathrm{H}$, Bussad WO. Elementos de Amostragem. $1^{\mathrm{a}}$ ed. São Paulo: Edgard Blucher; 2005.

10. Ponce MAZ, Wysocki AD, Scatolin BE, Andrade RLP, Arakawa $T$, Ruffino Netto $A$ et. al. Diagnóstico da tuberculose: desempenho do primeiro serviço de saúde procurado em São José do Rio Preto, São Paulo, Brasill. Cad Saude Publica [Internet]. 2013 [acesso em: 30 set 2014];29(5): 945-54. Disponível em: http://dx.doi.org/10.1590/S0102-

311X2013000500012.

11. Wysocki AD, Ponce MAZ, Scatolin BE, Andrade RLP, Vendramini SHF, Ruffino Netto $A$ et al. Delay in seeking initial care for Tuberculosis Diagnosis. Rev Esc Enferm USP [Internet]. 2013 [acesso em: 30 set 2014];47(2):440-7. Disponível em: http://dx.doi.org/10.1590/S0080-62342013000200024.

12. Scatena LM, Villa TCS, Ruffino Netto A, Kritski AL, Figueiredo TMRM, Vendramini SHF, et al. Difficulties in the accessibility to health services for tuberculosis diagnosis in Brazilian municipalities. Rev Saude Publica [Internet]. 2009 [acesso em: 30 set 2014];43(3):389-97. Disponível em:

http://dx.doi.org/10.1590/S0034-89102009005000022.

13. Sreeramareddy CT, Panduru KV, Menten J, Van den Ende J.

Time delays in diagnosis of pulmonary tuberculosis: a systematic review of literature. BMC Infect Dis [Internet]. 2009 [acesso em: 30 set 2014];9:91. Disponível em:

http://dx.doi.org/10.1186/1471-2334-9-91.

14. Scalco SV, Lacerda JT, Calvo MCM. Modelo para avaliação da gestão de recursos humanos em saúde. Cad Saude Publica [Internet]. 2010 [acesso em: 30 set 2014];26(3):603-14. Disponível em: http://dx.doi.org/10.1590/S0102-

311X2010000300017.

15. Oliveira MF, Arcêncio RA, Ruffino-Netto A, Scatena LM, Palha $\mathrm{PF}$, Villa TCS. A porta de entrada para o diagnóstico da tuberculose no Sistema de Saúde de Ribeirão Preto/SP. Rev Esc Enferm USP [Internet]. 2011 [acesso em: 30 set 2014];45(4):898-904. Disponível em:

http://dx.doi.org/10.1590/S0080-62342011000400015. 16. Barbosa NB, Elias PEM. As organizações sociais de saúde como forma de gestão público/privado. Cien Saude Colet [Internet]. 2010 [acesso em: 30 set 2014];15(5):2483-95. Disponível em: http://dx.doi.org/10.1590/S141381232010000500023.

17. Varella TC, Pieratoni CR. Mercado de trabalho: revendo conceitos e aproximando o campo da saúde. A década de $90 \mathrm{em}$ destaque. Physis [Internet]. 2008 [acesso em: 30 set 2014];18(13):521-44. Disponível em:

http://dx.doi.org/10.1590/S0103-73312008000300009.

18. Modesto P. Contrato de gestão no interior da organização administrativa como contrato de autonomia. Revista Eletrônica sobre a Reforma do Estado [Internet]. 2010 [acesso em: 30 set 2014];(23):1-30. Disponível em:

http://www.academia.edu/1063377/CONTRATO_DE_GESTAO_N O_INTERIOR_DA_ORGANIZACAO_ADMINISTRATIVA_COMO_CON TRATO_DE_AUTONOMIA.

19. Conselho Nacional de Secretários de Saúde. Nota Técnica de 22 de agosto de 2006. As Organizações Sociais como alternativa de gerência para estabelecimentos públicos de saúde. Brasília: CONASS; 2006

20. Tibério AA, Souza EM, Sarti FM. Considerações sobre avaliação de estabelecimentos de saúde sob gestão de OSS: o caso do Hospital Geral do Grajaú. Saude soc. [Internet]. 2010 [acesso em: 30 set 2014];19(3):557-68. Disponível em: http://dx.doi.org/10.1590/S0104-12902010000300008. 21. Monroe AA, Gonzáles RIC, Palha PF, Sassaki CM, Ruffino Netto A, Vendramini SHF et al. Envolvimento de equipes da atenção básica à saúde no controle da tuberculose. Rev Esc
Enferm USP [Internet]. 2008 [acesso em: 30 set 2014];42(2):262-7. Disponível em: http://dx.doi.org/10.1590/S0080-62342008000200008. 22. Santos MLSG, Villa TCS, Vendramini SHF, Gonzáles RIC, Palha PF, Santos NSGM et al. A gerência das ações de controle da tuberculose em municípios prioritários do interior paulista. Texto Contexto Enferm [Internet]. 2010 [acesso em: 30 set 2014];19(1):64-9. Disponível em: http://dx.doi.org/10.1590/S0104-07072010000100007. 23. Batista KBC, Gonçalves OSJ. Formação dos profissionais de saúde para o SUS: significado e cuidado. Saude soc. [Internet]. 2011 [acesso em: 30 set 2014]; 20(4):884-99. Disponível em: http://dx.doi.org/10.1590/S0104-12902011000400007.

24. Ministério da Saúde. Manual de recomendações para o controle da tuberculose no Brasil [Internet] Brasília: Ministério da Saúde; 2011 [acesso em: 30 set 2014]. Disponível em: http://www.cve.saude.sp.gov.br/htm/TB/mat_tec/manuais/MS11 Manual_Recom.pdf.

25. O'Dwyer G. A gestão da atenção às urgências e o protagonismo federal. Cien Saude Colet [Internet]. 2010 [acesso em: 30 set 2014];15(5):2395-404. Disponível em: http://dx.doi.org/10.1590/S1413-81232010000500014.

Artigo recebido em 03/12/2012.

Aprovado para publicação em 11/03/2014.

Artigo publicado em 30/09/2014. 